\title{
Acute neonatal airway management - The role of laryngeal mask airway
}

\author{
P M C Nair ${ }^{1}$, Anagha Padmarajan ${ }^{2}$ \\ From ${ }^{1}$ Emeritus Professor SAT, Goverment Medical College, ${ }^{2}$ Anaesthesia Resident, KIMS Health, Trivandrum, Kerala, India.
}

\begin{abstract}
Positive pressure ventilation with Ambu bag and face masks or T-piece resuscitation in preterm babies have proved their worth in acute neonatal airway management. However, failure rates are high and endotracheal intubation is the final life-saving procedure. However, intubation is an invasive procedure and requires good expertise, especially for handling acute emergency situations. Expertise of trainees as well as consultants is coming down rapidly due to various reasons. In these situations, supraglottic airways (laryngeal mask airway or LMA) seem to be ideal. Here, we are reviewing the application and utility of LMA in acute neonatal airway management.
\end{abstract}

Key words: Acute neonatal airway management, Supraglottic airway, Laryngeal mask airway or LMA

$\mathrm{T}$ he ability to maintain a patent airway and provide effective positive pressure ventilation (PPV) is the main objective of neonatal resuscitation. This is currently achieved using a face mask or an endotracheal tube. Both of these devices have major limitations and require adequate operator skills. In acute profound neonatal asphyxia, endotracheal intubation is the emergency life-saving procedure, however, it invites several complications. In certain situations, both face mask ventilation and tracheal intubation may prove difficult to establish, especially in patients with congenital abnormalities of the upper airway.

The expertise of Neonatal doctors doing emergency intubation is steadily falling due to a number of reasons [1]. With improvement in technology and advanced perinatal management, the number of asphyxiated newborns is less. Infants born through meconium-stained amniotic fluid (MSAF) are no longer routinely intubated. Most preterm babies are now stabilized on non-invasive respiratory support and early extubation is favored. Real-life intubation opportunities are continuing to fall. Mannequin use and lot of e-simulation exercises are going on but their clinical use is still far away and falls short at replicating secretions, airway instability/pressure, and edema. Stress and time pressure added to the fear of physiological instability of the newborn that has even worsen the situation. Clinicians are becoming less proficient in intubation and are very stressful for clinicians expected to intubate but not feeling competent to do so. Prolonged or multiple intubation attempts are more frequent, which in turn might prolong asphyxia, leading to intraventricular hemorrhage (IVH), oral, pharyngeal, oesophageal and airway injury.

\section{Access this article online}

Received - 30 August 2021

Initial Review - 16 September 2021

Accepted - 24 September 2021

DOI: $10.32677 / \mathrm{IJCH} .2021 . v 08.110 .3094$
PROMISING TOOLS FOR VISUALIZATION AND MANAGEMENT OF AIRWAYS

Videolaryngoscopy (VL) [2-4] is a promising tool that helps to visualize the airway, recognize the anatomy, and maintains the view while inserting endotracheal tube (ETT), confirming correct placement and securing the ETT. First attempt intubation success rate is better with VL. But intubating with a VL is a different experience and is ideal to perform in a controlled/supervised environment which will not be possible in an emergency situation. Furthermore, it is very expensive in the Indian scenario.

Over the past years, proponents of the laryngeal mask airway (LMA) device have suggested that its use could circumvent the problems of insufficient face mask ventilation and endotracheal tube placement.

Supraglottic airway devices (SADs) [5-11] have revolutionized the airway management. Currently, supraglottic airway or the laryngeal mask airway (LMA) is the most promising option and alternative to intubation. It is also effective and easy to use even in neonates. In 1981, Dr. Archie Brain designed the laryngeal mask airway (LMA) with the aim of producing an airway device that would be more practical than the face mask and less invasive than the tracheal tube [5]. The first successful supraglottic airway device, the LMA-Classic, became available in 1989. The functional elegance of the LMA is that it forms a low pressure airtight seal against the glottis rather than plugging the pharynx, thus combining ease of insertion and adequate airway patency. The inflatable cuff, sits in the hypopharynx, just above the glottic opening, and forms a low-pressure seal with surrounding tissues, channeling gases towards the larynx. Popularity of LMA in neonatology is rising gradually. Licensed LMA can be used for

Correspondence to: Dr. P M C Nair, Chaithanya, TC 25/1847, Thampanoor, Trivandrum - 695 001, Kerala, India. E-mail: drpmc50@gmail.com

(C) 2021 Creative Commons Attribution-NonCommercial 4.0 International License (CC BY-NC-ND 4.0). 
neonates $>2 \mathrm{~kg}$ and 34 weeks gestation. Smaller sizes are not yet available. International resuscitation guidelines now recommend their use from $1.5 \mathrm{~kg}$ body wt. There are published reports of randomized control trials upto $1 \mathrm{~kg}$ wt.

Indications for LMA in newborns [5]: Neonates who are late preterm, full-term newborns, newborns with birth weight $>2000 \mathrm{~g}$ (sometimes $>1500 \mathrm{~g}$ ) are observed with the following indications: (1) Cannot intubate and cannot ventilate, (2) Failure of face mask, (3) Maxilla-facial anomalies or injuries such as congenital anomalies involving the mouth, lip, tongue, palate, and neck, (4) Small mandible or large tongue.

\section{Classic LMA (cLMA) [5-12]}

cLMA (Fig. 1) is a first-generation reusable SAD. It is made of medical-grade silicone and contains no latex. It has a curved airway tube (shaft) connected to an elliptical spoon-shaped mask (cup) at a $30^{\circ}$ angle. There are two flexible vertical bars (aperture bars) to prevent the tube from being obstructed by the epiglottis. The inner rim of the mask is surrounded by an inflatable cuff. An inflation tube and self-sealing pilot balloon are attached to the proximal wider end of the mask. A black line runs longitudinally along the posterior aspect of the tube and it helps to detect any subsequent rotation of the mask on the tube axis. At the machine end of the tube is a $15-\mathrm{mm}$ connector. cLMA is available in 8 sizes, size 1 is used in neonates weighing $2.5-5 \mathrm{~kg}$. It has been postulated that a smaller size $(0.5)$ could be useful in preterm newborns. However, there are reports of successful use of size 1 in preterm neonates weighing $0.8-1.5 \mathrm{~kg}$.

\section{Insertion of cLMA}

Pre use check

As the device is reusable (up-to 40 times) and subject to wear and tear, before use it should be checked for damage. The airway tube should also be checked by flexing to ensure it does not occlude when bent. Finally, the lumen of the device must be checked to exclude the presence of foreign bodies introduced during the cleaning/sterilization process. Before insertion, the concave part of the mask is pressed against a hard surface while deflating it,

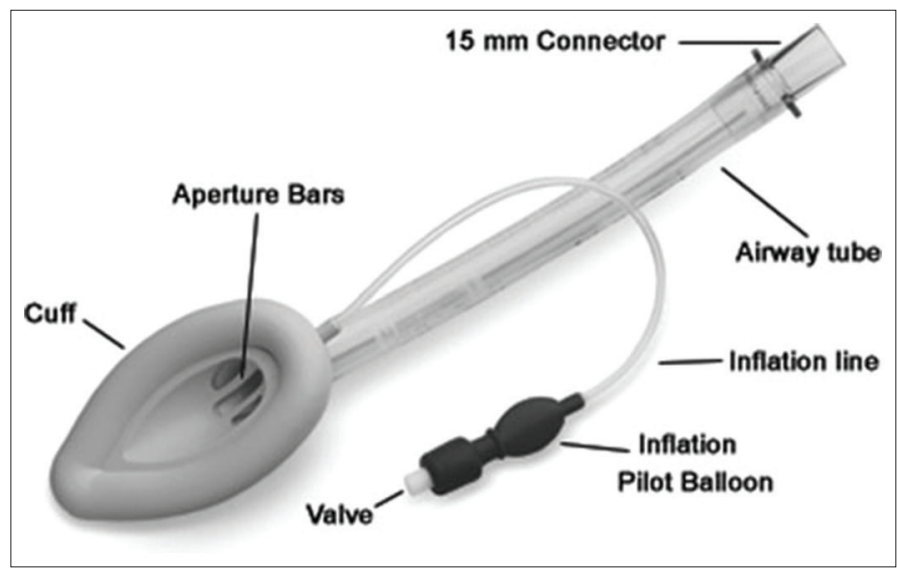

Figure 1: Classic LMA which causes the cuff to retract backward behind the bowl. The back of the mask is lubricated with a water-based gel.

\section{Standard insertion}

The head and neck of the neonates are placed in the sniffing position. LMA is grasped like a pen in the dominant hand. The tip of the operator's gloved index finger is placed at the junction of the tube and mask whilst the non-dominant hand maintains the position of the head and neck by cradling the occiput. The mask is inserted into the mouth and the bowl is kept pressed against the hard palate as it is advanced in one smooth movement into the hypopharynx. The hard and then the soft palate and finally posterior pharyngeal wall act as a scoop to guide the mask into place and prevent snagging on the tongue or epiglottis. The mask is advanced until resistance is felt. Without holding the tube, the cuff is then inflated with air. A maximum of $4 \mathrm{ml}$ air should be used to inflate the cuff of a size 1, LMA. The cuff should never be inflated with more than the recommended amount of air.

\section{Confirmation of correct placement}

When appropriately positioned the distal tip of the silicone cuff rests against the upper oesophageal sphincter, the sides of the cuff in pyriform fossa, and the upper part of the cuff against the tongue base. When the LMA cuff is inflated four observations assist confirmation of correct placement. (1) As the mask tip inflates, the LMA rises $0.5-2 \mathrm{~cm}$ before coming to an abrupt halt, (2) The anterior neck is seen to slightly fill, (3) The longitudinal black line running along the dorsal aspect of the tube should remain in the anatomical midline, (4) Bilateral chest expansion on ventilation.

Modifications to the original LMA [13-15] include ports for nasogastric tubes, capnography (end-tidal $\mathrm{CO}_{2}$ monitoring), alterations to the cuff shape to prevent gastric insufflation, and port to facilitate trans-LMA intubation by fiber-optic bronchoscope, etc.

\section{Proseal LMA [13-15]}

Proseal LMA (Fig. 2) is a second-generation SADs with minimal risk of aspiration and regurgitation. It was introduced in 2000. It has four main parts: the cuff, inflation line with pilot balloon, airway tube, and drain (gastric access) tube. All components are made from silicone and are latex-free. It is available in six sizes. Size 1 is used for neonate. It is wire reinforced, which makes it more flexible. There is a locating strap on the anterior distal tube to prevent the finger slipping off the tube and to provide an insertion slot for the introducer tool. The drain tube runs parallel and lateral to the airway tube until it enters the cuff bowl, where it continues to an opening in the tip. It is designed to prevent the epiglottis from occluding the airway tube. The integral bite block constitutes more than $1 / 3^{\text {rd }}$ of the shaft of whole sizes of LMA Proseal except size 1, where its only $1 / 6^{\text {th }}$ of the shaft length.

\section{The i-gel [13-15]}

Of the 7 brands of neonatal LMA studied (Fig. 3), the i-gel was found to be the easiest to use. The i-gel is an LMA with a 
solid cuff which, with body heat, molds to the shape of the airway. There were no insertion failures, ensuring lowest leak while delivering the highest peak inspiratory and expiratory pressures. Cases of successful LMA placement in neonates at the first attempt was reported between $69 \%$ and $100 \%$. LMA insertion technique is easily learnt and no sedation is required. The i-gel is a novel single use, cuffless SAD made of a medical-grade elastomer gel (styrene ethylene butadiene styrene). It has a short wide-bore airway tube with no grilles, an elliptical-shaped stem, an anatomically shaped bowl, an integral bite block, and a drain

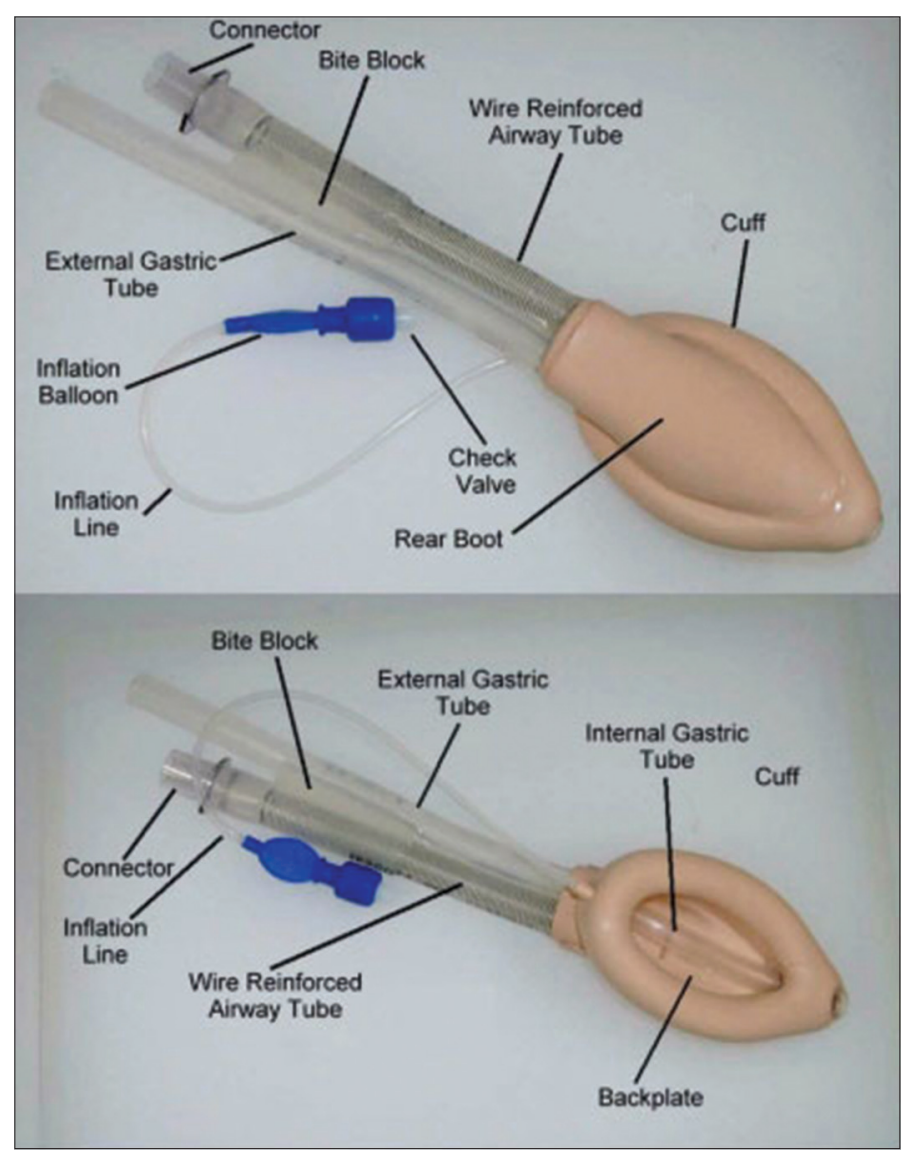

Figure 2: Proseal LMA tube. Size 1 i-gel lacks the drain tube. The i-gel is inserted in the sniffing position and after lubrication of the back, front, and sides of the device. The i-gel offers the potential for improved ease of use, improved ventilation, and increased safety compared to the cLMA, in a disposable SAD.

\section{Care and cleaning}

The reusable LMA should be gently cleaned with warm water and a dilute ( $8-10 \%)$ sodium bicarbonate solution until all visible material has been removed. A pipe cleaner-type brush should be inserted through the distal aperture to clean out the shaft, taking care not to damage the bars. The drain tube of the LMA-ProSeal should be cleaned carefully because it could be damaged by a stiff brush. The inflation valve should not be exposed to any cleaning solution, because this may cause valve failure. The LMA should be rinsed with tap water to remove residue and then dried and placed in a pouch. Autoclaving an LMA with water in the cuff may cause irreversible damage, so do not allow water to enter the cuff. The cuff should be emptied with the cuff uppermost and manually squeeze, to remove the fluid. Air should be removed from the cuff before autoclaving, as the residual air can expand in the heat and may damage the cuff, valve, or pilot balloon. The LMA can be autoclaved at temperatures up to $135^{\circ} \mathrm{C}$. The LMA should be allowed to cool to room temperature after sterilization. Chemical agents such as glutaraldehyde, phenol-based cleaners, iodine-containing cleaners, quaternary ammonium compounds, or ethylene oxide should not be used to clean or sterilize the LMA, since they get adsorbed onto the silicone and can cause pharyngitis and laryngitis as well as shorten the LMA life.

\section{ADVANTAGES AND DISADVANTAGES OF LMA Advantages of the LMA Over the Face Mask}

Bag-mask ventilation is a technique requiring a high and continuous level of training. Incorrect positioning of the face mask can cause gas leakage around the rim, and the use of excessive pressure may result in injuries to facial soft tissues. Laboratory studies have shown that most devices commonly used for positive

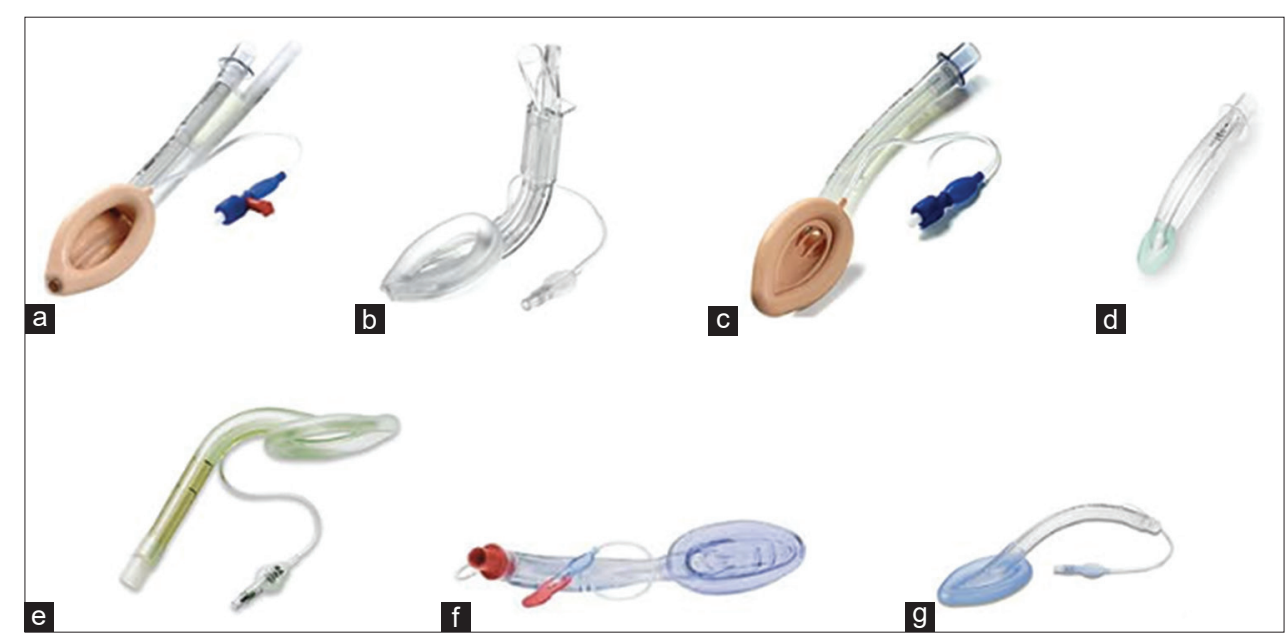

Figure 3: Types of LMAs used in neonates (a) LMA Proseal, (b) LMA Supreme, (c) LMA Classic, (d) i-gel, (e) Ambu ${ }^{\circledR}$ AuraOnce, (f) Air-Q, (g) Shiley 
pressure ventilation (PPV) with bag and mask may be unable to provide adequate tidal volumes during neonatal resuscitation. It has also been shown that the efficacy of bag-mask ventilation depends on the operator's skill [15].

The LMA offers many advantages over the face mask. Its use does not require manipulation of the patient's head, neck, and jaw. It also avoids compression of facial nerves. After positioning, the LMA is quite stable and frees the operator's hands for other important tasks. A better airtight seal is achieved with the LMA, providing more effective PPV. Its insertion technique, stable positioning, and function is not influenced by anatomical factors, that may make face mask ventilation difficult, especially in cases of congenital pathologies such as PierreRobin and Treacher Collins syndrome in which more expertise is required. In a meta-analysis including 52 randomized prospective trials, the advantages of the LMA over the face mask included: easier placement by inexperienced personnel; improved oxygen saturation; less hand fatigue, achieve and maintain effective PPV with the LMA compared with the face mask. It has been shown that the incidence of hypoxia is lower in infants with the use of the LMA than with the face mask.

\section{Advantages of the LMA Over Endotracheal Intubation [16]}

The LMA is less invasive and avoids laryngoscopy and all of its related adverse effects. In comparison with the endotracheal tube, the patient is subjected to a lower hemodynamic stress response during LMA positioning and removal. This could theoretically reduce the incidence of cerebral hemorrhage in neonates. In addition, tracheal edema, which may be caused by tracheal intubation, is avoided using the LMA. Its use may be lifesaving in patients with malformations of the upper airway when tracheal intubation and mask ventilation fail. Whereas successful intubation may require more than one attempt, the LMA can be easily inserted by non-anesthetist personnel. LMA insertion does not require the use of neuromuscular blocking agents, further diminishing pharmacological risk in neonates.

In summary, the advantages of the LMA over the tracheal tube include, increased speed and ease of placement by anesthetists, as well as non-anesthetist medical personnel, and trained nonmedical personnel, improved hemodynamic stability during insertion, minimal increase in intraocular pressure after insertion, and improved oxygen saturation.

\section{Disadvantages of the LMA [16]}

The potential disadvantages of the LMA include the following.

1. Gastric insufflation and aspiration, gastric distention, and vomiting: The LMA does not separate the respiratory and alimentary tracts, thus exposing the patient to the risk of aspiration and gastric insufflation during PPV; this fact may limit the efficacy of ventilation. Meticulous attention to the insertion and fixation of the LMA and avoidance of excessive positive pressure may eliminate this problem.

2. Inadequate alveolar ventilation: The LMA cuff forms a low-pressure seal against the larynx. The maximum seal pressure is $20-25 \mathrm{~cm} \mathrm{H}_{2} \mathrm{O}$. It is known that, during the first breaths at birth, pressures required to achieve effective ventilation can be higher, and in these cases the LMA may be ineffective. The recently introduced LMA-ProSeal, may alleviate this problem.

3. Impossibility of suctioning the airway: Depressed neonates with meconium aspiration syndrome require tracheal intubation and suctioning. The latter cannot be performed by means of the LMA, as the passage of suctioning tubes may be partially or totally impaired or cause laryngeal spasm.

4. Improper positioning and local soft tissue trauma, pharyngeal and lingual edema, abrasions, mucosal injury, etc.

\section{POTENTIAL APPLICATIONS OF LMA}

1. In neonatal resuscitation [17-28]: Official Scientific Societies such as the International Liaison Committee on Resuscitation (ILCOR), American Academy of Pediatrics, and American Heart Association have stated that the LMA may be useful when face mask and endotracheal intubation fail, although they do not recommend its routine use because of the lack of clear scientific evidence.

Studies on the efficacy of ventilation by medical and paramedical personnel in neonatal training models have shown that the LMA allows a patent airway to be obtained in a shorter time than the endotracheal tube. They also show that the incidence of failure is lower with the LMA. The LMA has gained increasing popularity for resuscitation at least as an aid in cases of difficult airway management or failure of conventional maneuvers.

Although this device cannot be considered as a substitute for the endotracheal tube, it could play an ancillary role, particularly in situations where assistance to the asphyxiated neonate is offered by paramedical staff or doctors who may have difficulty maintaining a high level of skill.

Higher rate of successful resuscitation, higher 5' Apgar scores, and less NICU admissions were seen than infants resuscitated with a facemask $(P<0.05)$. The 2020 International Liaison Committee on Resuscitation (ILCOR) suggests LMA use where mask ventilation is not effective or intubation is unsuccessful or not feasible. 2021 European Resuscitation Council recommends the use of LMAs in infants $>1500 \mathrm{~g}$ and/or 34 weeks' gestational age where facemask ventilation is prolonged or unsuccessful or intubation is either unsuccessful or not feasible [29].

2. In neonatal intensive care for surfactant administration [29-31]: Recently, the feasibility and practicality of administering surfactant using the LMA in preterm infants with respiratory distress syndrome were studied. The data indicate that the LMA can be used as a conduit to obtain rapid and non-invasive access to the trachea of preterm infants to administer surfactant. This would encourage the policy of "minimal handling" and could avoid respiratory and extra-respiratory complications 
such as stress and intraventricular hemorrhage associated with the tracheal intubation. Brimacombe et al. in 2004, used LMA for surfactant delivery [22]. A case series by Trevisanuto et al. [23] describes eight infants weighing 880-2520 $\mathrm{g}$ who received surfactant via LMA and were found to have significantly higher mean arterial-alveolar oxygen ratio $3 \mathrm{~h}$ later $(\mathrm{p}<0.01)$. In a recent narrative review, Roberts et al. [29] describes several case reports and six Randomized controlled trial (RCTs) of 357 infants and found the LMA to be a simple and effective mode of surfactant administration that could be used widely by clinicians of varying experience but called for further RCTs to determine their safety and effectiveness in both tertiary and non-tertiary settings before introduction into routine clinical practice. The largest RCT to date is by Roberts et al. [29] who randomized 103 infants 28-35 weeks' gestation and $>1250 \mathrm{~g}$ to surfactant via LMA or continuous positive airway pressure and found a significant decrease in the intubation rates in the LMA surfactant group, with no adverse events $(P=0.006$, Number Needed to Treat (NNT) $=4)$. Recent UK case series in $1.2 \mathrm{~kg}$ infants who received LMA surfactant showed $83 \%$ resolution of respiratory distress syndrome (RDS) and avoided intubation. In all cases, LMA insertion was successful in $78 \%$ of cases on first attempt and in $98 \%$ of cases on the first two attempts, similar in all levels of clinician.

3. LMA for neonatal difficult airways and transport $[32,33]$ : The LMA has also been used in neonates affected by malformations of the upper airway in emergency situations and during laryngoscopy and bronchial endoscopy. In particular, the LMA has proved useful in the intubation maneuver. LMAs have been used successfully during both land and air neonatal support.

4. In congenital anomalies of the face or injury. Neonates affected by multiple congenital arthrogryposis and micrognathia, and patients with bronchopulmonary dysplasia or undergoing cryotherapy for retinopathy of the prematurity have been ventilated with the LMA.

Longest reported duration of ventilation through LMA in a neonate is 8 days in a newborn with airway obstruction and severe Treacher Collins syndrome. Theoretically, the LMA may have a role in the weaning of patients with good pulmonary compliance. The efficacy of the LMA has also been reported in a case in which ventilation of a neonate with face mask had failed during helicopter transportation [33].

\section{Current Limitations}

The following includes few limitations of LMA:

1. Neonatologists to be trained to be proficient in LMA use.

2. Device suitable for even the tiniest babies are needed. Now size 1 can be used only in neonates weighing from $1500 \mathrm{~g}$ and above.

3. LMAs yet to be evaluated in preterm infant stabilization where obstruction at glottic level can frequently occur.

\section{CONCLUSION}

As endotracheal intubation competency and success rates are low and falling for pediatric and neonatal trainees, LMAs will play a major role as a promising alternative to intubation for ventilation or giving surfactant. Smaller LMAs are urgently required.

Training must be imparted to trainees and doctors for the use of LMA. In developing countries, cost may be high for initial purchase, but compared to portex endotracheal tube and also the complications taken into account, LMAs will be cost-effective and user friendly and can be reused about 40 times after sterilization.

\section{REFERENCES}

1. O'Shea JE, Scrivens A, Edwards G, Roehr CC. Safe emergency neonatal airway management: current challenges and potential approaches. Arch Dis Child Fetal Neonatal Ed 2021;2021;319398.

2. O'Shea J, Kirolos S, Thio M, Kamlin CO, Davis PG. Neonatal videolaryngoscopy as a teaching aid: The trainees' perspective. Arch Dis Child Fetal Neonatal Ed 2021;106:168-1.

3. Balaban O, Tobias JD. Videolaryngoscopy in neonates, infants, and children. Pediatr Crit Care Med 2017;5:477-85.

4. Lingappan K, Arnold JL, fernandes CJ, Pammi M. Videolaryngoscopy versus direct laryngoscopy for tracheal intubation in neonates.Cochrane Database Syst Rev 2018;6:CD009975.

5. Jones JR. Laryngeal mask airway: An alternative for the difficult airway. AANA J 1995;63:444-9.

6. Miller DM. A proposed classification and scoring system for supraglottic sealing airways: A brief review. Anesth Analg 2004;99:1553-9.

7. Wanous AA, Wey A, Rudser KD, Roberts KD. Feasibility of laryngeal mask airway device placement in neonates. Neonatology 2017;111:222-7.

8. Trevisanuto D, Micaglio M, FerrareseP, Zanardo V. The laryngeal mask airway: Potential applications in neonates archives of disease in childhood. Fetal Neonatal Ed 2004;89:F485-9.

9. Ramesh S, Jayanthi R. Supraglottic airway devices in children. Indian J Anaesth 2011;55:476-48.

10. White C, Cook TM, Peter A. Stoddart, a critique of elective pediatric supraglottic airway devices. Pediatr Anesth 2009;19:55-65.

11. Sinha A, Sharma B, Sood J. Pressure vs. Volume control ventilation: Effects on gastric insufflation with size-1 LMA. Pediatr Anesth 2010;20:1111-7.

12. Kundra P, Deepak R, Ravishankar M. Laryngeal mask insertion in children: A rational approach. Paediatr Anaesth 2003;13:685-90.

13. Bimla S, Chand S, Abhijit B, Kumra VP, Jayashree S. Proseal laryngeal mask airway-a study of 100 consecutive cases of laparoscopic surgery. Indian J Anaesth 2003;47:467-72.

14. Lopez-Gill M, Brimacombe J. The ProSeal laryngeal mask airway in children. Pediatr Anaesth 2005;15:229-34.

15. Goldmann K, Roettger C, Wulf H. Use of the ProSeal TM laryngeal mask airway for pressure-controlled ventilation with and without positive endexpiratory pressure in paediatric patients: A randomized, controlled study. Br J Anaesth 2005;95:831.

16. Sinha A, Sharma B, Sood J. ProSeal as an alternative to endotracheal intubation in pediatric laparoscopy. Pediatr Anesth 2007;17:327-32.

17. Bansal SC, Caoci S, Dempsey E, Trevisanuto D, Roehr CC. The laryngeal mask airway and its use in neonatal resuscitation: A critical review of where we are in 2017/2018. Neonatology 2018;113:152-61.

18. Zanardo V, Simbi A, Micaglio M, Cavallin F, Tshilolo L, Trevisanuto D. Laryngeal mask airway for neonatal resuscitation in developing country: Evaluation of an educational intervention. Neonatal LMA: An educational intervention in DRC. BMC Health Serv Res 2010;10:254.

19. Grein AJ, Weiner GM. Laryngeal mask airway versus bag-mask ventilation or endotracheal intubation for neonatal resuscitation. Cochrane Database Syst Rev 2005;18:CD003314.

20. Micaglio M, Doglioni N, Parotto M, Zanardo V, Ori C, Trevisanuto D. Training for neonatal resuscitation with the laryngeal mask airway: A comparison of the LMA-ProSeal and the LMA-classic in an airway management manikin. Pediatr Anaesth 2006;16:1028-31. 
21. Trevisanuto D, Parotto M, Doglioni N, Ori C, Zanardo V, Micaglio M. The supreme laryngeal mask airway TM (LMA): A new neonatal supraglottic device: Comparison with Classic and ProSeal LMA in a manikin. Resuscitation 2012;83:97-100.

22. Gandini D, Brimacombe J. Manikin training for neonatal resuscitation with the laryngeal mask airway. Pediatr Anaesth 2004;14:493-4.

23. Trevisanuto D, Micaglio M, Pitton M, Magarotto M, Piva D, Zanardo V. Laryngeal mask airway: Is the management of neonates requiring positive pressure ventilation at birth changing? Resuscitation 2004;62:151-7.

24. Zanardo V. Neonatal resuscitation by laryngealmask airway after elective caesarean section. Fetal Diagn Ther 2004;19:228-31.

25. Zanardo V, Weiner G, Micaglio M, Doglioni N, Buzzacchero R, Trevisanuto D. Delivery room resuscitation of near-term infants: Role of the laryngeal mask airway. Resuscitation 2010;81:327-30.

26. Schmölzer GM, Agarwal M, Kamlin CO, Davis PG. Supraglottic airway devices during neonatal resuscitation: An historical perspective, systematic review and meta-analysis of available clinical trials. Resuscitation 2013;84:722-30.

27. Zhu XY, Lin BC, Zhang QS, Ye HM, Yu RJ. A prospective evaluation of the efficacy of the laryngeal mask airway during neonatal resuscitation. Resuscitation 2011;82:1405-9.

28. Madar J, Roehr CC, Ainsworth S, Ersdal H, Morley C, Rüdiger M, et al. European resuscitation council guidelines 2021: Newborn resuscitation and support of transition of infants at birth. Resuscitation 2021;161:291-326.
29. Roberts CT, Manley BJ, O'Shea JE, Stark M, Andersen C, Davis PG, et al. Supraglottic airway devices for administration of surfactant to newborn infants with respiratory distress syndrome: A narrative review. Arch Dis Child Fetal Neonatal Ed 2021;106:336-41

30. Pinheiro JM, Santana-Rivas Q, Pezzano C. Randomized trial of laryngeal mask airway versus endotracheal intubation for surfactant delivery. J Perinatol 2016;36:196-201.

31. Roberts KD, Brown R, Lampland AL, Leone TA, Rudser KD. Laryngeal mask airway for surfactant administration in neonates: A randomised control trial, randomized controlled trial. J Pediat 2018;193:40-6.

32. Brimacombe J, Gandini D. Airway rescue and drug delivery in an $800 \mathrm{~g}$ neonate with the laryngeal mask airway. Paediatr Anaesth 1999;9:178

33. Wightman S, Godden C, O'Shea J. A review of the use of supraglottic airways in neonates for use during interhospital transfer. Early Hum Dev 2019;138:1048-55

Funding: None; Conflicts of Interest: None Stated.

How to cite this article: Nair PM, Padmarajan A. Acute neonatal airway management -the role of Laryngeal Mask Airway. Indian J Child Health. 2021; 8(10):343-348. 